\title{
Finding a Parsimonious Path for Primary Care Practice Transformation
}

\author{
Asaf Bitton, MD, MPH \\ Ariadne Labs, Brigham and Women's Hospital and the Harvard T. H. Chan School of Public Health, Boston, Massachusetts \\ Division of General Medicine, Brigham and Women's Hospital, Boston, Massachusetts \\ Center for Primary Care, Harvard Medical School, Boston, Massachusetts \\ Ann Fam Med 2018;16(Suppl 1)S16-S19. https://doi.org/10.1370/afm.2234.
}

W here do we stand in 2018 with primary care practice transformation in the United States? There is no longer much debate that primary care, and the neighborhood that supports or hinders it, needs to be substantially renovated to meet an array of systemic challenges. Many barriers remain to providing accessible, coordinated, comprehensive care over time to a defined panel of patients in an individualized manner. Clinicians are challenged by the ever-expanding nature of medical innovation and wide array of recommended care to be delivered. Fragmented information and organizational architectures often impede the central coordinating role that primary care must play for patients. Electronic health records (EHRs) are unfit for that purpose and often require spending twice as much time in documentation as in direct care. ${ }^{1}$ Payment systems atomize payment as units of in-person visits instead of care for people's ongoing total needs. Appropriate patient demand for increased access and nonvisit modes of communication furthers the time pressures. The primary care workforce contends with burnout and insufficient growth in the training pipeline. New and often external mandates for organizational change use up limited adaptive reserve.

Recognizing the need to find ways to adapt to meet these challenges quickly, clinicians, payers, and policy makers have directed unprecedented attention and resources into primary care transformation efforts during the past decade. Exponential early growth in prac-

Conflicts of interest: Bitton serves as a part-time senior advisor to the Comprehensive Primary Care Plus Initiative at the Center for Medicare and Medicaid Innovation.

\section{CORRESPONDING AUTHOR}

Asaf Bitton, MD, MPH

Ariadne Labs, 3rd Floor East

401 Park Dr

Boston, MA 02215

abitton@bwh.harvard.edu tice or payer efforts to implement the patient-centered medical home $(\mathrm{PCMH})$ have now morphed into larger regional and national efforts, often on a multipayer basis, to attempt to catalyze practice change on a larger and faster scale. Huge implementation science and health services research efforts and resources have been poured into not only understanding whether newer team-based models, such as PCMH, work but, more importantly, into how and in what settings different transformation elements can best flourish. These extra resources often come with added administrative and documentation requirements that can chew up the support, energy, and will for change. ${ }^{2}$

Into this arena the EvidenceNOW initiative arrives-the largest investment by the Agency for Healthcare Research and Quality (AHRQ) in understanding facilitators, barriers, and elements of successful primary care transformation in small and medium-size practices at a regional level to improve care around the core components (aspirin use, blood pressure control, cholesterol management, smoking cessation $[\mathrm{ABCS}]$ ) of heart health. The first articles to come out of this effort in this journal supplement help us to start to answer questions around the key challenges that primary care transformation initiatives must meet to succeed, and some of the best ways to do so.

None of the challenges described above is novel, and yet the EvidenceNOW research efforts not only document them on a wider scale, but also add important texture and new content at an important inflection point for US primary care. The articles in this supplement show that among small and medium-size practices, rates of major disruptive changes (personnel turnover or EHR adoption) can reach $35 \%$ of any given practices each year. By any measure, this almost predictable chaos ensures that practice capacities for meaningful and sustained organizational change are endemically limited. Unsurprisingly, the use of quality improvement strategies across these practices varies 
widely and suggests the need for ongoing facilitation to overcome frequent disruption and often unready environments for change. ${ }^{3}$

The EvidenceNOW articles thankfully go beyond problem identification; they point to important features of practice change that appear to be associated with higher levels of practice improvement in the change process. Notably, the design and theory of EvidenceNOW center on facilitated change implemented at a regional or community level through cooperatives, with sufficient timelines to embed sustainable change processes around a common set of important goals for cardiovascular disease prevention. Grounding the work in communities that are given the space and time to find consensus around the "best" changes to make together around high-value common targets was wise. Contrast this approach to the more common change models seen in primary care: often overspecified checklists of nonadapted changes targeted toward individual practices alone.

Furthermore, the EvidenceNOW work points to what I would term as valuable elements of "quality improvement hygiene." The articles point to many promising and evidence-based hygienic practices, including use of embedded registries, continuous review of electronic clinical quality measures, a regular space and time for practice team communication, community involvement in change, and budgeting enough around practice recruitment within cooperatives. They also suggest that there may be some areas of accepted wisdom in primary care transformation that may be more complex than first appears, or concepts for which further evidence is needed to understand their valence in small to medium-size practices. For example, although adaptive reserve for change is unquestionably important, in and of itself it may not be able to overcome other structural barriers to catalyzing practice performance improvement. ${ }^{4}$ Staff and clinicians appear to perceive practice adaptive reserve for change in different ways across even the same work environment. ${ }^{5}$ Clearly, segmenting an approach to reducing burnout and maintaining appetite and aptitude for change are necessary. And transforming with patient and community advisors embedded within every step of the process to guide and inform the work is a successful strategy for engagement and sustainability. ${ }^{6}$

The food writer Michael Pollan once famously condensed the nutrition field into a few simple rules for healthy eating. At a total of 7 words, their parsimonious insight and pithiness are memorable and accurate: "Eat food, not too much, mostly plants." I often use these words with my patients, with some subsequent adaptation from him (he now talks about eating "real food," not processed) and me (I added "exercise" for obvious reasons). So I end up with 4 adapted little rules, at 11 total words: "Eat real food, not too much, mostly plants, exercise a lot." It seems to me to be a reasonably accurate and terse summary of what we know about the evidence base for achieving and maintaining a healthy weight. Perhaps given the accumulated experience of practice facilitation, ${ }^{8}$ large multipayer initiatives, ${ }^{9}$ academic initiatives ${ }_{1}^{10}$ EvidenceNOW, and others, we might be ready, with all due requisite humility, for a parsimonious set of tenets for primary care practice transformation. These few rules, adapted by practices for use in their community context, could boil down to: "Build whole change together, pace yourself, focus on what matters most, practice quality improvement hygiene" (Table 1 ).

First, practices, as well as local, regional, and national systems trying to help primary care achieve its potential, must work on large-scale change bolistically so change can be successful. Practice leaders and systems supporters must look beyond incremental short-term fixes to focus on large-scale, long-term change to enable their staff to provide high-quality, relationshipfocused, coordinated care over time. Everyone in the practice should be enlisted in the effort, including patients, families, and communities. Change can happen more effectively when it is possible for whole communities, regions, or systems to move together. Finding sustainable financial models and structural support for this work is crucial, and the extension agent model used in EvidenceNOW, if supported financially, is one promising approach.

Second, practices need to pace themselves as they work on change. They must be careful to not work too fast on too many things at one time as they seek to make large-scale change over time. Staff and leaders must sequence the change, starting with leadership, practice culture, and empanelment as core foundations of transformation efforts. Then they can move to other features of team-based care, access, behavioral health integration, and coordination. As they change, they must continuously monitor and build adaptive reserve and work on maintaining joy of practice through the challenges of change. And practices, systems, and policy makers must allow enough time before passing judgment on the success of such endeavors.

Third, practices should focus on what matters most. They should explicitly target the most beneficial primary care outcomes or functions for patients and communities. These may include focusing improvements and capacity expansions in the following areas: building long-term trusting and healing relationships with patients and communities; risk-stratified care management for high-need patients; key chronic diseases, such as cardiovascular disease and diabetes; benefi- 
cial preventive measures (ie, immunizations, smoking cessation, colorectal cancer screening); integrated behavioral health; and social needs identification and community resource connections. There are many other good things that primary care can and, perhaps one day, should routinely do. But to achieve sustained success, practices must make hard choices to focus on the highest value areas, save the rest for another day, and work on what matters most now.

Fourth, staff and leaders must practice regular "quality improvement bygiene." They should meet routinely as an

\section{Table 1. A Few Parsimonious Enablers and Tenets of Primary Care Transformation}

\section{System-level enablers}

Payment reform

Practice facilitation

Data use and aggregation

More usable EHRs

Practice-level tenets

Build whole change together

Focus on large-scale, long-term change to become the practice that you want to be for your patients and your staff

Enlist everyone in the practice in the effort, including patients, families, and communities

Use facilitators and facilitation strategies

Change as a community, region, or system when possible

Find the right financial payment model and structural support for long-lasting change

Pace yourself

Do not work too fast on too many things, but also do not work only on marginal changes that do not matter

Sequence the change, starting with leadership, practice culture, and empanelment. Then move to other features of team-based care and coordination

Build adaptive reserve and work on maintaining joy of practice

Allow enough time to pass before passing judgment

Focus on what matters most

Explicitly target the most beneficial primary care outcomes or functions to for patients and communities, such as:

Building long-term relationships

Risk-stratified care management for high-need patients

Key chronic diseases such as cardiovascular disease and diabetes mellitus

Beneficial preventive measures (ie, immunizations, smoking cessation, colorectal cancer screening)

Integrated behavioral health

Social needs identification and resource connections

Save the rest for another day-work on what matters most now

Practice regular "quality improvement hygiene"

Meet regularly as an entire team to review change

Set targets and review progress toward those targets

Use registries of electronic clinical quality measures (do not wait for old and often inaccurate claims quality data)

Use an established quality improvement method-try changes and learn from them

Involve patients and your community in the change upfront and throughout

EHR = electronic health record entire team to review change, go over practice challenges, accelerate learning, and share complex patient cases, ideally once a week. They should set targets and review progress toward those targets as an entire team. Regular use of electronic clinical quality measures and registries will help to set and monitor attainment of goals - they must not wait for old and often inaccurate claims quality data. Using an established quality improvement method can build sequential improvement cycles - they should continue to try changes and learn from them regardless of success. And practices must involve patients and your community in the change upfront and throughout the transformation journey.

But practice-level strategies alone will not be sufficient alone to transform primary care-there are also at least 4 systemic enablers: facilitation, data aggregation, functional EHRs, and payment. The EvidenceNOW studies, along with accumulated transformation experience across the United States, suggest that sustained facilitation is a key enabler to practice and regional change. Although the best forms of facilitation are still debated, it is clear that for most practices, some type of facilitation support is useful. As more primary care practices change the way they provide care, finding the most cost-effective, unobtrusive forms of facilitation will increasingly become an important area of implementation research and improvement.

In addition, aggregating data for practices from the array of payers and clinicians in their region is an important enabler of target setting and monitoring. This aggregation of clinical data can happen at the practice level through the use of electronic clinical quality measures that abstract data directly from the EHR (instead of claims). For practices not in integrated systems, however, data aggregation should happen through payers or regional consortia with interoperable access to multiple sources of EHR and payer data so practices can have accurate, practice-wide views of clinical quality performance, utilization measures, and total cost of care.

EHR refinement must occur to improve these systems to better serve clinicians and patients. EHRs must be streamlined with better user interfaces and less cumbersome documentation mechanisms to reduce the time and burden associated with them. And they must be reimagined to fit better for their true purpose, which is to keep an accurate, accessible record of care over time that enhances patient-clinician interactions, not detracts from them. That means lessening their often obtuse complexity and moving them from primarily billing systems toward enabling platforms to engage patients and coordinate care.

Finally, payment systems-the major barrier perpetually threatening progress toward practice 
transformation-must be fundamentally reformed. It is common to declare that current fee-for-service payment in the United States is misaligned with primary care practice policy and transformation. Misaligned is a misnomer. Misalignment might imply that relatively modest redirection or amelioration might correct the problem, or that it is one of but many competing priorities for improvement. Fee-for-service payment for modern US primary care, however, is fundamentally at odds with attainment of core primary care functions for patients. There is simply no way that a modern primary care practice can achieve team-based care functions under current fee-for-service payment, or even marginal PCMH monthly additional payments so common across primary care. ${ }^{11,12}$ This view is more than opinion-it is mathematical reality. My colleagues and I have calculated that achieving team-based care functions requires major (greater than $60 \%$ ) capitated or episodic fees for most practices (greater than 95\%) to not lose money providing them. ${ }^{13}$ That is not to say that capitation would not be bringing its own well-documented challenges around a large panel size and the risk of excluding patients with complex or high-cost illnesses. Rather, it is to make clear the need for creating a fiscal space for changing practice using the tenets above.

Primary care clinicians need a payment system that supports and sustains their attempts at change, not one that impedes them through added fiscal risk and administrative burden. If there is one element that might have been missing from the EvidenceNOW cooperatives, it is in this realm of fundamental financial payment reform (not alignment). Although perhaps out of scope given AHRQ research funding and structure, future work must address the melding of transformative primary care payment reform to practice change. Primary care practices simply cannot be asked to improve care, avoid burnout, reduce costs, and achieve system targets while also wondering whether in so doing they will reduce their income. When they finally have the safe fiscal space for change, and take on the work through a few core tenets and enabling system features, we may finally have a pathway toward primary care sustainability and success.

To read or post commentaries in response to this article, see it online at http://www.AnnFamMed.org/content/16/Suppl_1/S16.
Key words: primary care; transformation; facilitation; implementation science; payment

Submitted February 18, 2018; submitted, revised, March 10, 2018; accepted March 11, 2018.

Funding support: Publication of this article was supported by the Agency for Healthcare Research and Quality (AHRQ) through contract No. HHSA290201200019l.

Disclaimer: This work represents the opinions of the authors and should not be interpreted as official positions of the Agency for Healthcare Research and Quality, the Center for Medicare and Medicaid Innovation, or the US Department of Health and Human Services.

\section{References}

1. Sinsky C, Colligan L, Li L, et al. Allocation of physician time in ambulatory practice: a time and motion study in 4 specialties. Ann Intern Med. 2016;165(11):753-760.

2. Bujold, E. When practice transformation impedes practice improvement. Ann Fam Med. 2015;13:273-275.

3. Balasubramanian B, Marino M, Cohen DJ, et al. Use of quality improvement strategies among small to medium-size US primary care practices. Ann Fam Med. 2018;16(Suppl 1):S35-S43.

4. Henderson K, DeWalt DA, Halladay J, et al. Organizational leadership and adaptive reserve in blood pressure control: the Heart Health NOW Study. Ann Fam Med. 2018;16(Suppl 1):S29-S34.

5. Cuellar A, Krist AH, Nichols LM, Kuzel AJ. Effect of practice ownership on work environment, learning culture, psychological safety, and burnout. Ann Fam Med. 2018;16(Suppl 1):S44-S51.

6. English AF, Dickinson LM, Zittleman L, et al. A community engagement method to design patient engagement materials for cardiovascular health. Ann Fam Med. 2018;16(Suppl 1):S58-S64.

7. Pollan M. In Defense of Food. New York, NY: Penguin Press; 2009.

8. Baskerville NB, Liddy C, Hogg W. Systematic review and metaanalysis of practice facilitation within primary care settings. Ann Fam Med. 2012;10(1):63-74.

9. Anglin G, Tu HA, Liao K, Sessums L, Taylor EF. Strengthening multipayer collaboration. Strengthening multipayer collaboration: lessons from the comprehensive primary care initiative. Milbank $Q$. 2017;95(3):602-633.

10. Koch U, Bitton A, Landon BE, Phillips RS. Transforming primary care practice and education: lessons from 6 academic learning collaboratives. J Ambul Care Manage. 2017;40(2):125-138.

11. Basu S, Phillips RS, Song Z, Landon BE, Bitton A. Effects of new funding models for patient-centered medical homes on primary care practice finances and services: results of a microsimulation model. Ann Fam Med. 2016;14(5):404-414.

12. Magill MK. Time to do the right thing: end fee-for-service for primary care. Ann Fam Med. 2016;14(5):400-401.

13. Basu S, Phillips RS, Song Z, Bitton A, Landon BE. High levels of capitation payments needed to shift primary care toward proactive team and nonvisit care. Health Aff (Millwood). 2017;36(9):1599-1605. 


\section{Get the Annals of Family Medicine by E-mail}

Make sure you see every new issue

while it's fresh; have the table of

contents sent to you by e-mail for

easy access to articles of interest.

Don't miss important research.

Request the e-mail table of contents at

http://www2.highroadsolution.com/

aafp_annals_preference_center/search.aspx

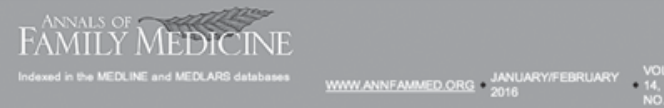

Now Available:

The Wonder and the Mystery

Annals anthology of personal reflections and innovative ideas

The fill text of the jourmal is availabie online at htro: aggregabors, including PubMed Central, EBSCC, and MDConsult. The Annafs is indexed in the
MEDLINE and MEDLARS, Science Citation Index Expanded, Current Contents Clinical Medidine, PSyclNFO, EMBASE, and CINHAL databases.

EDITORIALS

In This issue: Size Matters

Achieving PCMH Stans May Not Be Meaningtul for Small Practices

The Paradox of Size: How Small, Independent Practloes Can Thive in Value-Based Care

ORIGINAL RESEARCH

Solo and Small Practices: A Vital, Diverse Part of Primary Gare

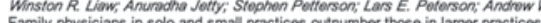

Large Indegendent Primay Care Medical Groups

Lawrence P. Casallino; Melinda A. Chen; C. Todd Staub; Matthew J. Press; Jayme L

Large physician-owned groups have the potential to make primary care attractive to physicians

Primary Care Physician Panel Size and Quality of Care: A Posulation-Based Study in Ontario. Simane Dahrouge; Wiwam Hogg; Jaime Younger, Elizaboth Muggah; Grant Russell; Richard H. Glazior
In Ontario, larger patient panel sizes do not decrease quality of care, but cancer screening rates are slightly lower.

Wilingness to Exchange Heatth Information via Mobile Devices: Findings From a

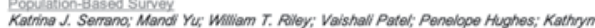
Marchesin', Audie A. Ationzs 